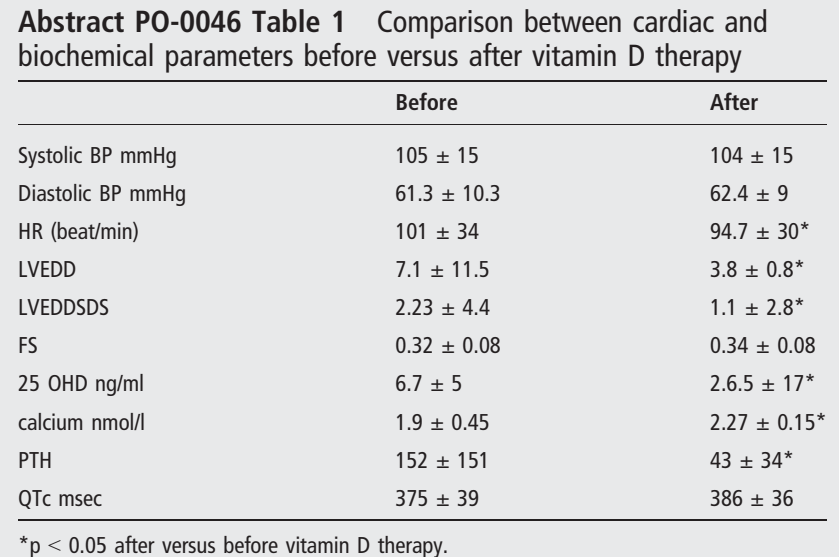

Discussion Improvement of the recorded changes in the heart rate and LVEDD after VD therapy implements an important role of VD through its effect on the concentration of the extracellular calcium ion that could modify the strength of the myocardial contraction through excitation-contraction coupling.

Conclusion These data strongly indicate that the maintenance of an optimal vitamin D status may be a promising approach for the prevention and/or therapy of myocardial diseases and in countries with high prevalence of VDD, vitamin D supplementation can prevent this risk of cardiac dysfunction.

\section{PO-0047 WITHDRAWN}

\section{PO-0048 WITHDRAWN}

\section{PO-0049 CORONARY ARTERY ANOMALIES IN PATIENTS UNDERGOING ARTERIAL SWITCH OPERATION}

${ }^{1} \mathrm{~V}$ Ziesenitz, ${ }^{1} \mathrm{M}$ Gorenflo, ${ }^{2} \mathrm{M}$ Karck, ${ }^{3} \mathrm{~T}$ Loukanov. ${ }^{1}$ Pediatric Cardiology \& Congenital Heart Diseases, Center for Childhood and Adolescent Medicine, Heidelberg, Germany; ${ }^{2}$ Department of Cardiac Surgery, Heidelberg, Germany; ${ }^{3}$ Section of Congenital Cardiac Surgery, Department of Cardiac Surgery, Heidelberg, Germany

\subsection{6/archdischild-2014-307384.722}

Background and aims The arterial switch operation (ASO) is the surgical standard of care for repair of transposition of the great arteries (D-TGA). Until recently, anatomical variations of the coronary arteries, especially the intramural course of a single coronary artery, were considered contraindications for ASO. Transfer of the coronary arteries may be a surgical challenge in these cases increasing the risk of (sub-)acute coronary artery occlusions.

Methods We report our management of two exemplary cases of D-TGA with coronary artery anomalies:

(1) Single coronary ostium of RCA, Cx and LAD originating from aortic sinus II and an intramural course of the proximal LAD.

(2) Side-by-side position of the great arteries, RCA and LAD originating from sinus I and Cx from sinus II.

Results Both neonates successfully underwent ASO with transfer of the coronary arteries.

During the post-operative period, patient 1 was diagnosed with a subacute anteroseptal ischemia and was then managed conservatively. Follow-up echocardiogram at 12 months demonstrated satisfactory left and good right ventricular function.

The postsurgical course of patient 2 was uneventful with good biventricular function at follow-up.

Conclusions Anatomical variations of the coronary arteries require adaptions of the surgical technique of coronary artery transfer. Nowadays, ASO is even possible in patients with D-TGA and complex coronary anomalies. The long-term management, however, has to be evaluated, e.g. regarding the need for coronary artery re-surgery.

\section{PO-0050 ABSENT PULMONARY VALVE IN A PATIENT WITH ALAGILLE SYNDROME}

${ }^{1}$ V Ziesenitz, ${ }^{1} \mathrm{D}$ Köhler, ${ }^{2} \mathrm{C}$ Gläser, ${ }^{3} \mathrm{~T}$ Loukanov, ${ }^{1} \mathrm{M}$ Gorenflo. ${ }^{1}$ Pediatric Cardiology and Congenital Heart Diseases, Center for Childhood and Adolescent Medicine, Heidelberg, Germany; ${ }^{2}$ Institute of Human Genetics and Medical Biology, Halle, Germany; ${ }^{3}$ Section of Congenital Cardiac Surgery, Department of Cardiac Surgery, Heidelberg, Germany

\subsection{6/archdischild-2014-307384.723}

Background and aims Absent pulmonary valve (APV) is a rare congenital defect of the right ventricular outflow tract (RVOT). The genetics of APV are unknown. However, mutations in the NOTCH-signalling pathway have been associated with RVOT obstruction. Mutations in the $J A G-1$ gene cause a broad spectrum of symptoms, ranging from an isolated heart defect to the complete clinical features of Alagille syndrome.

We present the case of a 14 month-old girl with APV and a family history of Alagille syndrome.

Methods Pulmonary stenosis and a large ventricular septal defect (VSD) had been diagnosed prenatally. Postnatal echocardiogram revealed an APV, pulmonary stenosis, a large sub-aortal VSD, and right ventricular hypertrophy.

Genetic analysis of the $J A G-1$ gene showed a frame-shiftmutation in exon 12 of the JAG-1 gene that had not been described before.

The patient underwent corrective heart surgery at 9 months of age. The VSD and the native pulmonary artery orifice were closed surgically. A valved xenograft conduit (Contegra ${ }^{\circledR}, 14$ $\mathrm{mm}$ ) was implanted between the RV and the pulmonary artery. Results The last follow-up echocardiogram at 12 months of age demonstrated a sufficient pulmonary valve, closed VSD, resolving right ventricular hypertrophy and good biventricular function.

Conclusions Genetic mutations affecting the NOTCH-signalling pathway can be involved in the pathogenesis of APV. Therefore it is essential to characterise patients with NOTCH-signalling pathway defects by their clinical features and by the underlying mutations in order to develop future therapeutic approaches of APV.

\section{Endocrinology/Diabetes/Metabolism}

\section{PO-0051 ZINC AND COPPER DISORDERS IN CHILDREN WITH DIABETES TYPE 1}

${ }^{1} \mathrm{M}$ Smahi, ${ }^{2} \mathrm{M}$ Aribi, ${ }^{2} \mathrm{~L}$ Hamouda, ${ }^{2} \mathrm{~L}$ Labadi, ${ }^{2} \mathrm{~L}$ Ysmaildahlok, ${ }^{3} \mathrm{~S}$ Bendeddeouche. ${ }^{1}$ Pediatrics and Neonatology, Mother-Child University Hospital Tlemcen, Tlemcen, Algeria; 'University of Tlemcen, Laboratory of Applied Molecular Biology and Immunology, Tlemcen, Algeria; ${ }^{3}$ University of Tlemcen, Department of Pediatrics, Tlemcen, Algeria

10.1136/archdischild-2014-307384.724 